\title{
27. On the Influence of the Atmospheric Dust on the Zodiacal Light Polarization
}

\author{
N. B. Divari and S. N. Krylova \\ Odessa Polytechnic Institute \\ Odessa, U.S.S.R.
}

\begin{abstract}
Three-color observations of the nightglow brightness and polarization were made at the Majaky Observatory in October 1969 to determine the brightness of zodiacal light at the ecliptic pole. On the assumption that the degree of polarization of zodiacal light is 0.20 at the ecliptic pole, the corresponding brightnesses are found to be 68, 70, and 96 G2 stars of the 10th magnitude per square deg. at $0.87,0.53$, and $0.58 \mu$.

The large diurnal variations in observed intensity and direction of polarization are attributed to the effects of dust in the Earth's atmosphere.
\end{abstract}

$\mathrm{F}_{\mathrm{p}}$ OR INVESTIGation of the zodiacal light component in the brightness of the night sky at the ecliptic pole, photoelectric observations of the night sky light were made at the observatory Majaky near Odessa (USSR) in autumn 1969. The observation was carried out with interference filters $\lambda=0.37,0.53$, and $0.58 \mu$ and a Polaroid which allows measurement of intensity, degree and angle of polarization by Fessenkov's (1935) method. At the time of these observations the zenith distance of the North ecliptic pole varied from $26^{\circ}$ to $65^{\circ}$. The observed brightnesses were expressed in units of the stars G2, of the 10th magnitude per square deg.

Figure 1 shows for the North ecliptic pole the observed brighcness $I_{\text {obs, }}$, the degree of polarization $P$ (in percent) and the angle $\varphi$ of polarization measured from the meridian of the ecliptic pole in a counterclockwise direction. The dashed line shows the angle $\beta_{\odot}$ between the meridian of the ecliptic pole and the direction from the ecliptic pole to the Sun.

The measured degree of polarization is equal to 2 to 3 percent. The degrees of polarization averaged over all the nights of observation are equal to
0.020 for $\lambda=0.37 \mu, 0.031$ for $\lambda=0.53 \mu$ and 0.026 for $\lambda=0.58 \mu$.

The observed intensity of polarized component of light $\left(I_{\mathrm{obs}}\right)_{p}$ is determined by the formula

$$
\left(I_{\mathrm{obs}}\right)_{p}=I_{\mathrm{obs}} P
$$

where $I_{\text {obs }}$ is the observed intensity of light, $P$ is the observed degree of polarization. The intensity of the polarized component $I_{p}$ outside the atmosphere may be calculated by the formula

$$
I_{p}=\left(I_{\mathrm{obs}}\right)_{p} p^{-\sec z}
$$

where $p$ is the transmission coefficient of the atmosphere and $z$ is the zenith distance of the ecliptic pole.

The values $I_{p}$ averaged over every night of observation are listed in table 1. The figures in parentheses give the intensity averaged over the measurements made when the zenith distance of the ecliptic pole was lower than $60^{\circ}$.

In table 2 our values are compared with the data obtained by other authors quoted in the references.

If all the polarization was caused only by the scattering of sunlight by the zodiacal cloud, the 

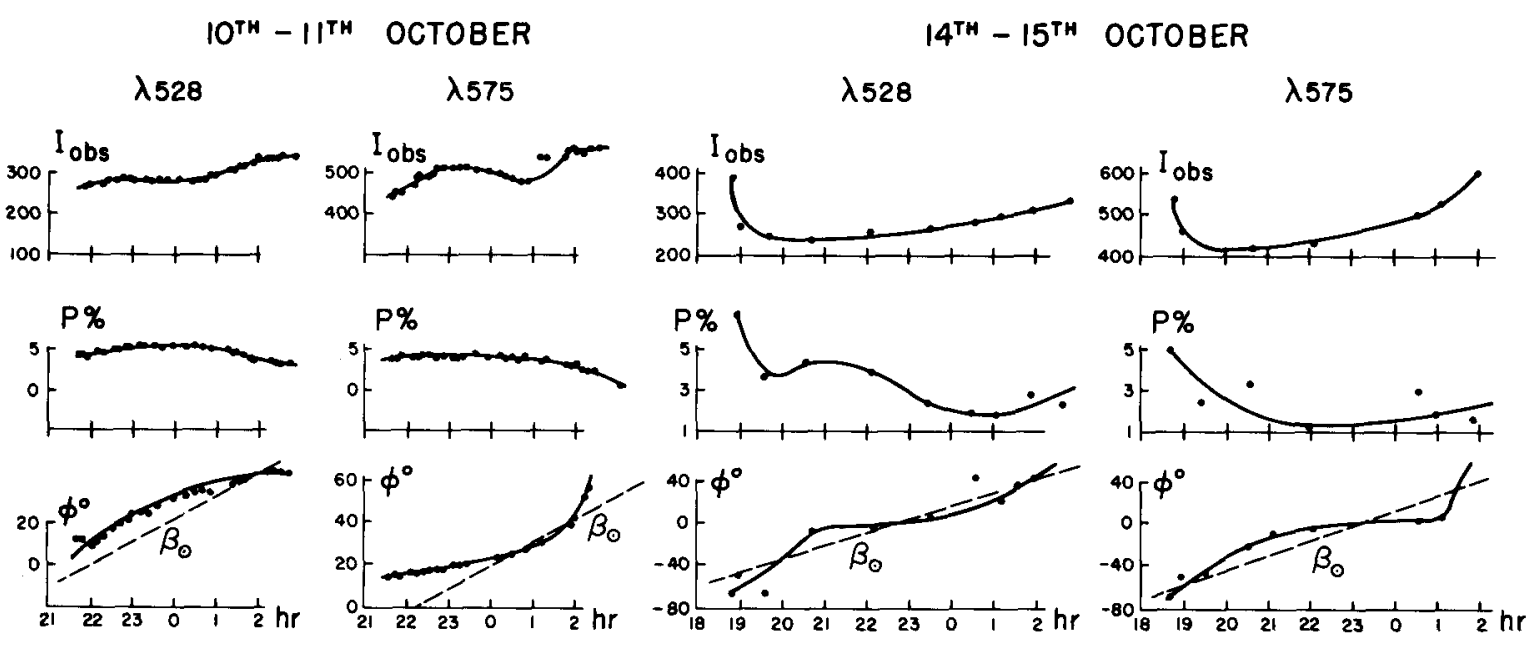

$13^{\text {TH }}-14^{\text {TH }}$ OCTOBER

$16^{\text {TH }}-17^{\text {TH }}$ OCTOBER
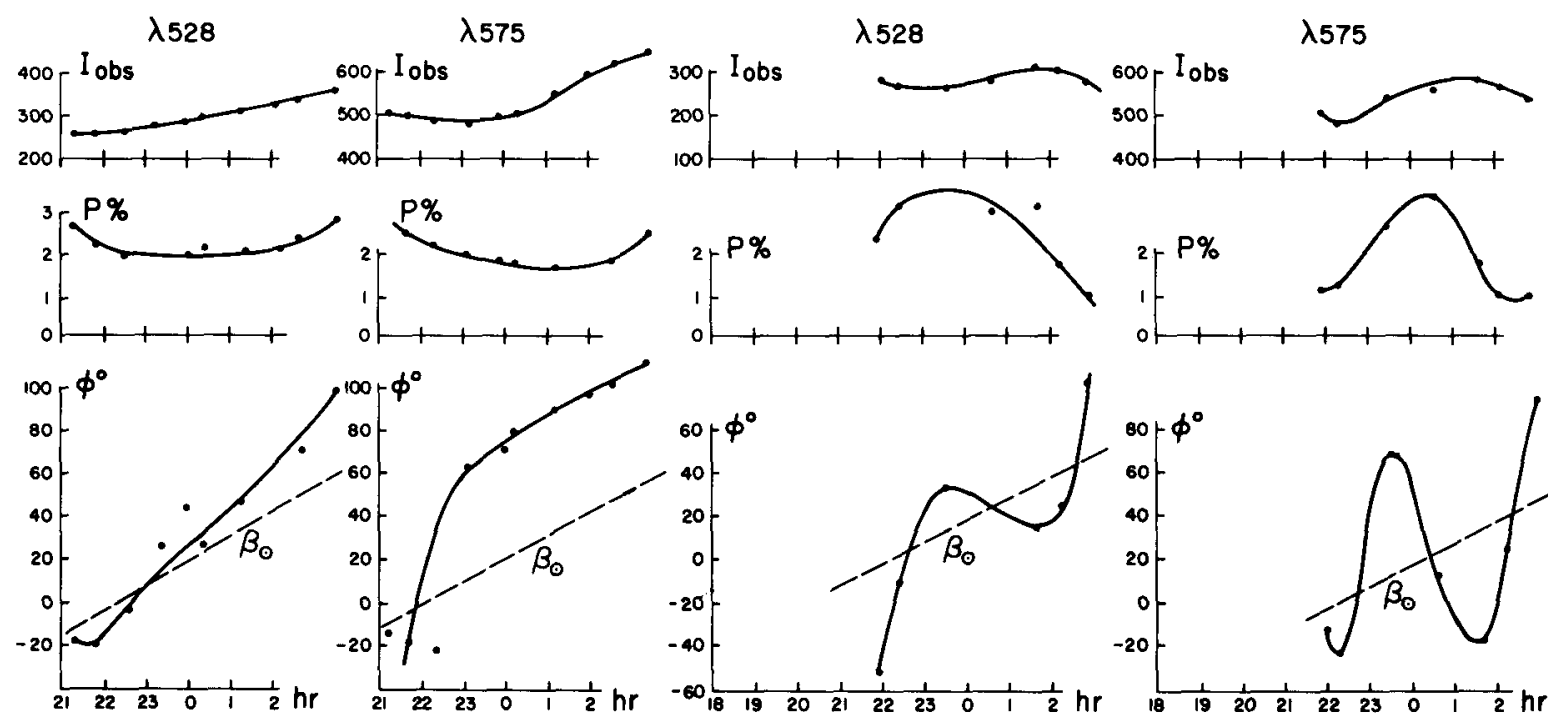

Figure 1.-Measured intensity $I_{\text {obs, }}$ observed degree $P$ (in percent) and observed angle $\phi$ of polarization in the North ecliptic pole.

intensity of zodiacal light $I_{z L}$ at the ecliptic pole would be determined from the values $I_{p}$ and $P_{z L}$ by the formula

$$
I_{Z L}=I_{p} / P_{Z L}
$$

If, according to Weinberg (1964), we adopt $P_{Z L}=$ 0.20 , we get from our values of $I_{p}$ the following intensity of zodiacal light at the ecliptic pole:

$I_{Z L}=71$ for $\lambda 0.53 \mu$ and $I_{Z L}=103$ for $\lambda 0.58 \mu$

But the observed polarization is not induced by the zodiacal light only, because the direction of the measured polarization does not coincide with the direction to the Sun. As may be seen in figure 1, the angle $\varphi$ differs considerably from the angle $\beta_{\odot}$. The deviation of the polarization angle from the scattering angle was observed in the zodiacal light by J. L. Weinberg (1964) in the light of the day sky (the sunlight scattered by the atmosphere) by E. V. Pyaskovskaya-Fessenkova (1958) and in the twilight by G. V. Rosenberg (1942). A qualitative explanation of this phenomenon as the result of multiple scattering and reflection from the Earth surface may be found in 
TABLE 1.-The Averaged Values of $\mathrm{I}_{\mathrm{p}}$ in Units of the Stars G2 of the 10th Magnitude per Square Deg

\begin{tabular}{lll}
\hline \multicolumn{1}{c}{ Night } & $\lambda=0.53 \mu$ & $\lambda=0.58 \mu$ \\
\hline Oct. 10-11, 1969 & $18.8(19.3)$ & $26.1(27.5)$ \\
Oct. 13-14, 1969 & $12.0(9.3)$ & $17.8(16.0)$ \\
Oct. 14-15, 1969 & $11.3(11.0)$ & $22.1(17.0)$ \\
Oct. 16-17, 1969 & $15.0(16.0)$ & $16.3(18.0)$ \\
& $-14.3(13.9)$ & $20.6(19.6)$ \\
\hline
\end{tabular}

TABLe 2.-Values of $\mathrm{I}_{\mathrm{p}}$ Resulting From Different Investigations

\begin{tabular}{lc}
\hline \multicolumn{1}{c}{ Reference } & $I_{p}$ \\
\hline Weinberg (1963) & 18.5 \\
Beggs et al. (1964) & 35 \\
Dumont (1965) & 12.6 \\
Dumont and Sanchez (1966) & 9.7 \\
Gillet (1967) & 11.5 \\
Wolstencroft and Rose (1967) & 26.5 \\
Sparrow and Ney (1968) & 10.5 \\
Ingham and Jameson (1968) & 10.7 \\
Present study " & 14.3 \\
\multicolumn{1}{l}{ " } & 20.6 \\
\hline
\end{tabular}

the paper of H. C. van de Hulst (1948). In a paper by Divari (1968), it was pointed out that scattering of light in the Earth's atmosphere (the tropospheric component) may have an influence on the degree and on the angle of observed polarization.

To estimate this influence, we have analyzed our observations at the ecliptic pole. The reduction of the observations was made by the method of successive approximations. Let us suppose that measured intensity $I_{\text {obs }}$ consists of five components, that is

$$
I_{\mathrm{obs}}=I_{z L} p^{\mathrm{sec} z}+I_{S} p^{\mathrm{sec} z}+I_{N}+I^{\prime}{ }_{z L}+I^{\prime}{ }_{S}
$$

where $I_{Z L}$ and $I_{S}$ are the intensities of the zodiacal light and all the stars outside the atmosphere, $I_{N}$ is the intensity of the light of the night sky, $I_{Z L}^{\prime}$ and $I_{S}^{\prime}$ are the intensity of the zodiacal light $I_{Z L}$ and the starlight $I_{S}$ scattered by the atmosphere. The intensity of tropospheric components $I^{\prime}{ }_{Z L}$ and $I_{S}^{\prime}$, the degrees of polarization $P^{\prime}{ }_{Z L}$ and $P^{\prime}{ }_{S}$ and the angles of polarization $\beta^{\prime}{ }_{Z L}$ and $\beta_{S}^{\prime}$ of these tropospheric components may be computed by the formulae given in Divari's (1968) paper.
As a first approximation we have adopted two models of the zodiacal light: one according to Megill and Roach (1961) with intensity at the ecliptic pole equal to 110 stars, and the other according to Divari (1968) with intensity at the ecliptic pole equal to zero. For these two models we have computed the tropospheric components $I_{Z L}^{\prime}$ and $I^{\prime} s$ by the formulae given by Divari (1968). The tropospheric brightnesses $I_{z L}^{\prime}$ and $I_{S}^{\prime}$ obtained in such a way were small as compared to the observed brightness for the two models. Taking these computed quantities $I_{S}, I^{\prime}{ }_{S}$ and $I_{Z L}, I^{\prime}{ }_{Z L}$ (for the two models) and subtracting them from $I_{\mathrm{obs}}$, we obtained the intensity $I_{N}$ of the light of the night sky (atmospheric component). We have used this intensity $I_{N}$ as a first approximation for the reduction of the measured brightnesses $I_{1}, I_{2}$ and $I_{3}$ at three positions of the Polaroid. Subtracting $I_{N}$, $I_{S}, I_{S}^{\prime}, I_{Z L}^{\prime}$ from the measured brightnesses $I_{1}, I_{2}$, $I_{3}$ we can obtain the intensity $\left(I_{Z L}\right)_{1},\left(I_{Z L}\right)_{2}$ and $\left(I_{Z L}\right)_{3}$ of the zodiacal light by only three positions of the Polaroid, turned by steps of $60^{\circ}$. These reduced values of $\left(I_{Z L}\right)_{1},\left(I_{Z L}\right)_{2},\left(I_{Z L}\right)_{3}$ were used for determining the degree and angle of the observed zodiacal light.

The averaged values of the degree of polarization for three wavelengths and two models of the zodiacal light are listed in table 3.

Table 3.-The Averaged Degree of Polarization $\mathrm{P}_{\mathrm{ZL}}$ at the Ecliptic Pole

\begin{tabular}{|c|c|c|c|c|c|c|}
\hline \multirow[b]{2}{*}{$\lambda, \mu$} & \multicolumn{3}{|c|}{$\begin{array}{l}\text { First model of the } \\
\text { zodiacal light }\end{array}$} & \multicolumn{3}{|c|}{$\begin{array}{l}\text { Second model of the } \\
\text { zodiacal light }\end{array}$} \\
\hline & 0.37 & 0.53 & 0.58 & 0.37 & 0.53 & 0.58 \\
\hline$P_{Z L}$ & 0.075 & 0.08 & 0.11 & 0.31 & 0.475 & 0.057 \\
\hline
\end{tabular}

One can see that for the first model the degree of polarization at the ecliptic pole is very low $(\sim 0.10)$ and for the second model it is very high $(\sim 0.45)$. This shows that the brightness $I_{N}$ of atmospheric component found in the first approximation does not provide the required degree of polarization for the zodiacal light $(\sim 0.20)$ at the ecliptic pole. To have that value of the degree of polarization, we have to choose such a value of the intensity $I_{N}$ of the atmospheric component that, being subtracted from the measured intensity $I_{1}, I_{2}, I_{3}$, it would provide a degree of polarization 
TABLE 4.-The Averaged Brightnesses of the Zodiacal Light at the Ecliptic Pole (for $\mathrm{z} \leq 60^{\circ}$ and $\mathrm{P}_{\mathrm{ZL}}=0.20$ ) Outside the Atmosphere in Units of Stars G2 10th Magnitude per Square Deg.

\begin{tabular}{lccc} 
Night & $\lambda=0.37 \mu$ & $\lambda=0.53 \mu$ & $\lambda=0.58 \mu$ \\
\hline $\begin{array}{l}\text { Oct. 10-11, 1969 } \\
\text { Oct. 13-14, 1969 }\end{array}$ & -70 & 95 & 127 \\
$\begin{array}{l}\text { Oct. 14-15, 1969 } \\
\text { Oct. 16-17, 1969 }\end{array}$ & 50 & 55 & 84 \\
$\begin{array}{c}\text { Averaged } \\
\text { over all } \\
\text { the nights }\end{array}$ & 68 & 78 & 84 \\
& & 70 & 96 \\
\hline
\end{tabular}

equal to 0.20 . The intensity $I_{N}$ found in such a manner together with $I_{S}, I_{S}^{\prime}$ and $I^{\prime}{ }_{2 L}$ found above were used for the determination of the intensity $I_{Z L}$ of the zodiacal light according to formula (4). The values obtained for the brightness of the zodiacal light at the ecliptic pole are listed in table 4 .

As is seen from that table, the intensity of the zodiacal light at the ecliptic pole increases with the increase of the wavelength $\lambda$.

As the observed direction of polarization does not coincide with the reference plane, we have computed the intensity and angle of polarization of the unknown component which turns the polarization vector out from this plane. To achieve this, we have taken for the polarized component of the zodiacal light the following values: $\left(I_{p}\right) \lambda_{0.53}=70 \times 0.20=14$ and $\left(I_{p}\right) \lambda_{0.58}=96 \times 0.20=$ 19 stars $\mathrm{G} 2$ of the 10th magnitude per square deg.

In figure 2 three vectors are shown, representing three components of the light of the night sky at the ecliptic pole: $\left(I_{Z L}\right)_{p}$ is the zodiacal light component directed to the Sun and $\left(I_{\mathrm{obs}}\right)_{p}$ is the observed polarized intensity (observed angle $\varphi$ ) and an unknown component $\left(I_{2}\right)_{p}$ with an unknown angle $\psi$.

Projecting the vectors on the direction forming an angle of $45^{\circ}$ with the vector $\left(I_{2}\right)_{p}$ and taking into account the necessity to double the angles,

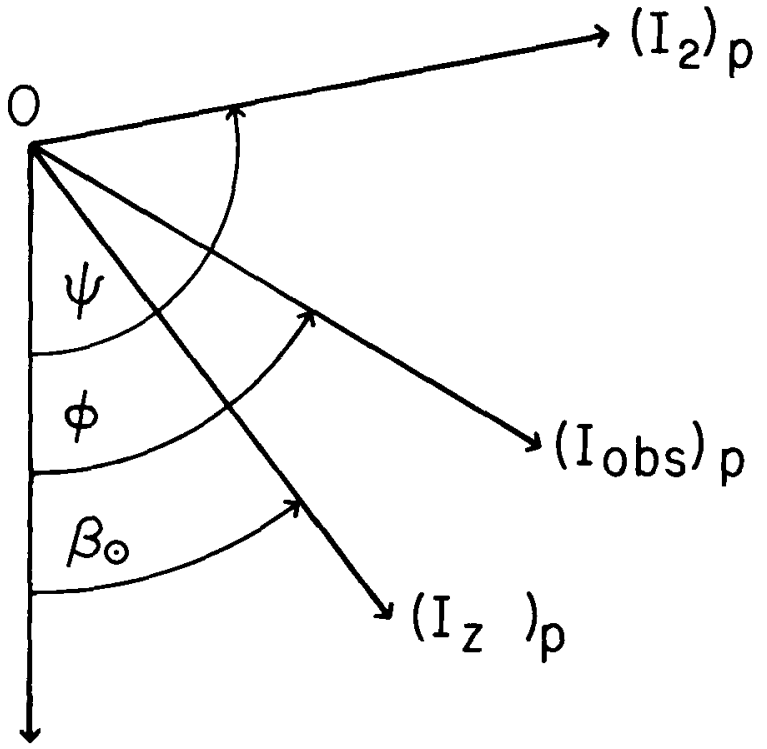

FIGURE 2.-Diagram of the vectors of polarization: $\left(I_{\mathrm{obs}}\right)_{p}$ is the observed vector of polarization with angle; $\left(I_{Z L}\right)_{p}$ is the vector of the zodiacal light polarization with angle; $\left(I_{2}\right)_{p}$ is the polarization vector of an unknown component.

we have

$$
\tan 2 \psi=\frac{\left(I_{\mathrm{obs}}\right)_{p} \sin 2 \varphi-\left(I_{z L}\right)_{p} \sin 2 \beta_{\odot}}{\left(I_{\mathrm{obs}}\right)_{p} \cos 2 \varphi-\left(I_{Z L}\right)_{p} \cos 2 \beta_{\odot}}
$$

If we project the three vectors on the direction forming $45^{\circ}$ with vector $\left(I_{Z L}\right)_{p}$, we have

$$
\left(I_{2}\right)_{p}=\left(I_{\text {obs }}\right)_{p} \frac{\sin 2\left(\varphi-\beta_{\odot}\right)}{\sin 2\left(\psi-\beta_{\odot}\right)}
$$

We have computed $\left(I_{2}\right)_{p}$ and $\psi$ according to formulae (5) and (6). Figure 3 shows these two quantities. As may be seen from this figure, the intensity and angle of polarization of the unknown component vary through the night in a wide range. These results show that Stokes parameters of the zodiacal light are very much disturbed by the Earth's atmosphere and their determination is possible only in very good atmospheric conditions. 
$10^{T H}-11^{T H}$ OCTOBER
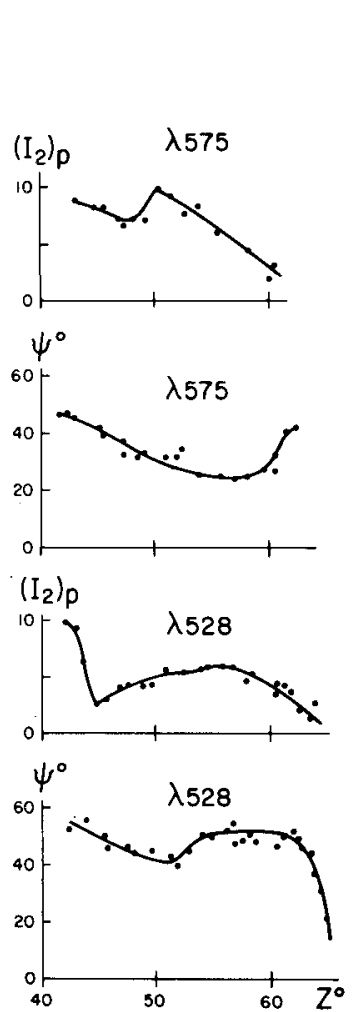

$13^{\text {TH }}-14^{\text {TH }}$ OCTOBER

$14^{\text {TH }}-15^{\text {TH }}$ OCTOBER

$16^{\mathrm{TH}}-17^{\mathrm{TH}}$ OCTOBER
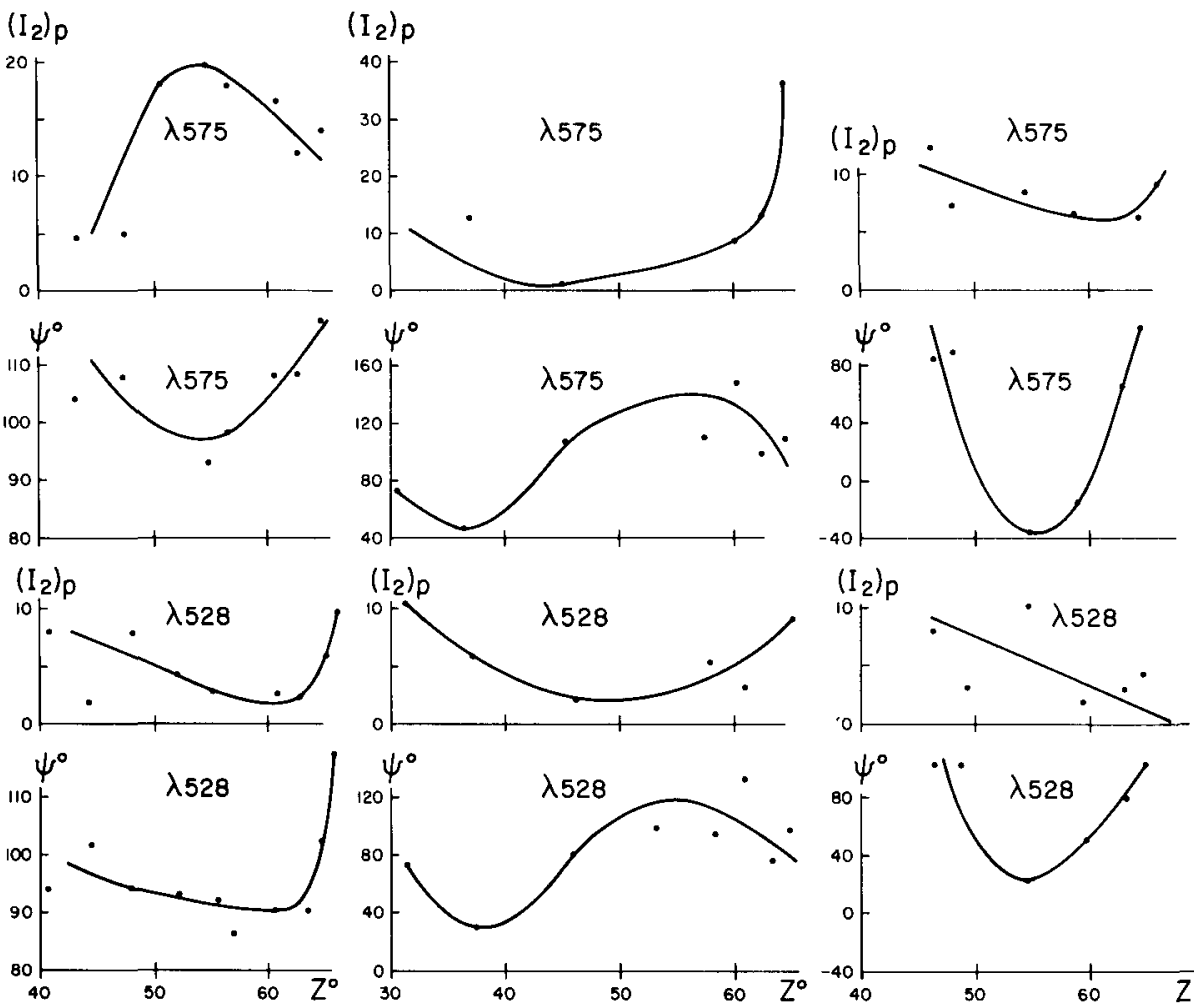

Figure 3.-The intensity $\left(I_{2}\right)_{p}$ and the angle $\psi$ of the polarized unknown component.

\section{REFERENCES}

Beggs, D. W., Blackwell, D. E., Dewhirst, D. W., and Worstencroft, R. D., 1964. Further observations of the zodiacal light from a high altitude station and investigation of the interplanetary plasma. III. Photoelectric measurements of polarization, Monthly Notices Roy. Astron. Soc., 128, 419-430.

Divari, N. B, On the polarization of light scattered by the troposphere of several components of the night sky, Astron. Zhurn. Akad. Nauk., SSSR, 45, 634-644.

Dumont, R., 1965. Separation des composantes atmospherique, interplanetaire et stellaire du ciel nocturne a $5000 \AA$. Application a la photometrie de la lumiere zodiacale et du gegenschein, Ann. d'Astrophys., 28, 265-320. Translation in NASA TT F-11, Dec. 1967, 164.

Dumont, R., AND Sanchez, M. F., 1966. Polarisation du ciel et polarisation de la lumiere zodiacale vers $5000 \AA$, sur l'ensemble de la sphere celeste, Ann. d'Astrophys., 29, 113-118.

Fessenkov, V. G., 1935. Determination de la polarisation de la couronne solaire, Astron. Zhurn. Akad. Nauk., SSSR, 12, 309-321.

Gillet, F. C., 1967. Measurement of the brightness and polarization of zodiacal light from balloons and satellites, in The Zodiacal Light and Interplanetary Medium, edited by J. L. Weinberg, NASA SP-150, Supt. of Documents, U.S. Govt. Printing Office, Washington.

INGHAM, M. F., AND JAMESON, R. F., 1968. Observations of the polarization of the night sky and a model of the zodiacal cloud normal to the ecliptic plane, Mon. Not. Roy. Astron. Soc., 140, 473-482.

Megild, L. R., AND Roach, F. E., 1961. The integrated starlight over the sky, National Bureau of Standards Tech. Note No. 106.

Pyaskovskaya-Fessenkova, E. V., 1958. Scattering and polarization of light by the atmosphere in the Libyan Desert, Akademii Nauk. Doklady, SSSR, 123, 269-271. 
Rosenberg, G. V., 1942. On a new phenomenon in the scattered light of a twilight sky, Akademii Nauk. Doklady. SSSR, 36, 270-274.

Sparrow, J. G., And Nex, E. P., 1968. OSO-B2 satellite observations of zodiacal light, Astrophys. $J ., 154,783-787$.

van DE Hulst, H. C., 1948. The Atmospheres of the Earth and Planets, edited by G. P. Kuiper, Chicago.

Weinberg, J. L., 1963. Photoelectric Polarimetry of the Zodiacal Light at $\lambda$ 5300, thesis, Colorado.

,- 1964 . The zodiacal light at $5300 \AA$, Ann. d'Astrophys., 27, 718-738.

Wolstencroft, R. D., AND Rose, L. J., 1967. Observations of the zodiacal light from a sounding rocket, Astrophys. J., 147, 271-292. 